\title{
The Contributions of Whole-body Magnetic Resonance Imaging for the Diagnosis and Management of Chronic Recurrent Multifocal Osteomyelitis
}

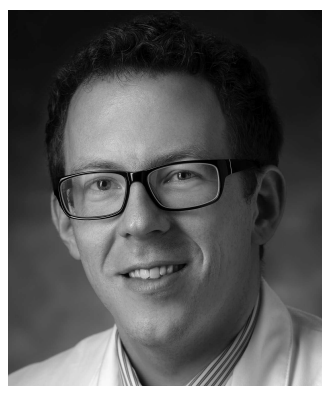

A form of the disease today known as chronic recurrent multifocal osteomyelitis (CRMO) was described in 1972 by Giedion, et al as "Subacute and chronic symmetrical osteomyelitis." "The authors described the findings of multiple symmetrical lesions in the metaphyses of bone that resembled infectious osteomyelitis; however, no organism could be isolated. More than 40 years later, their initial description, which may be summarized by chronicity, multifocality, symmetry, metaphyseal predilection, and lack of identification of an infectious organ remains remarkably accurate. The condition has been described with a multitude of different names; however, chronic recurrent multifocal osteomyelitis, which is documented as early as $1978^{2}$, remains commonly used.

Although CRMO appears to be diagnosed correctly with increasing frequency, the true prevalence is unknown. This may be due, at least in part, to the variability of clinical presentation. Signs and symptoms may be subtle, inflammatory markers may be normal, and osseous lesions may be clinically undetectable. A notorious characteristic of CRMO appears to be the discrepancy between a frequently underestimated burden of osseous inflammation based on clinical examination, laboratory inflammatory markers, and radiography, and the actual number of osseous inflammatory foci demonstrated on whole body imaging ${ }^{3}$.

Following the first study describing the usefulness of whole-body magnetic resonance imaging (WB-MRI) for the diagnosis of $\mathrm{CRMO}^{3}$, skeletal scintigraphy, which uses bone-seeking radioactive tracers, is less commonly used. WB-MRI has evolved into the imaging modality of choice at many institutions worldwide $e^{4,5,6,7}$ because the absence of ionizing radiation favorably complies with the "as low as reasonably possible" practice mandate and the accuracy for the demonstration of multifocality, symmetry, osseous inflammation, and bone marrow replacing processes. In addition, the superior soft tissue resolution of MRI affords the accurate characterizations of fascia, muscles, tendons, ligaments, vessels, and nerves, which can yield important information toward the differential diagnosis.

WB-MRI protocols typically consist of short-tau inversion recovery (STIR) and T1-weighted pulse sequences ${ }^{3,4}$. STIR pulse sequence types provide exquisite sensitivity for the detection of CRMO lesions through the use of robust fat suppression and high sensitivity for inflammatory tissue processes. STIR images can be thought of as inverted bone scan images. It is, however, important to remember that STIR signal hyperintensity is not specific to any process and can be seen with edema, hemorrhage, and cellular infiltration. T1-weighted sequences can be thought of as producing fat-sensitive images, which provide exquisite accuracy for the characterization of bone marrow. True bone marrow edema preserves the normal amounts of marrow fat and demonstrates homogeneously bright $\mathrm{T} 1$ signal intensity, whereas bone marrow replacing processes would be expected to cause a loss of the bright T1 signal of lesions that are hyperintense on STIR images.

Although CRMO lesions often present with a characteristic pattern consisting of periphyseal bone marrow edema pattern with a metaphyseal predilection, the appearance may not be characteristic enough to exclude conditions such as leukemia and lymphoma, histiocytoses, and multifocal osteosarcoma. In such cases, percutaneous core biopsy may still be required. Biopsy of CRMO lesions can be challenging, however, because lesions are often invisible on radiography and computed tomography ${ }^{3}$. Interventional MRI is an emerging technique that allows for the percutaneous osseous biopsy of CRMO lesions with high accuracy owing to its ability to directly visualize and target the lesion part with maximum signal abnormality ${ }^{8}$.

In addition to the contribution of helpful information for the diagnosis of CRMO, the radiation-free nature of WB-MRI renders this technique well suited for surveillance and monitoring of therapy response ${ }^{5}$. In this issue of The Journal, Viot, et al present longterm data on the clinical and

See WB-MRI followup in CRMO, page 1455

Personal non-commercial use only. The Journal of Rheumatology Copyright (c) 2015. All rights reserved. 
radiological activity of CRMO at or beyond 10 years after the initial diagnosis ${ }^{9}$. A reevaluation of 17 patients was conducted using physical examination to determine clinical symptoms, and WB-MRI to determine the presence of CRMO lesions. Limited comparison of WB-MRI data was made with whole-body bone scans that were obtained during initial work. Because there is no consensus about the optimal treatment of CRMO, therapy regimens were naturally highly variable among the study population. After re-evaluation, about $50 \%$ of patients were found to have symptomatic disease and were receiving treatment, whereas the other $50 \%$ of the study population was asymptomatic and did not receive treatment. In both patient groups, WB-MRI demonstrated inflammatory bone lesions characterized by STIR edema pattern, which suggests active inflammation.

New information that can be derived from this study is that regardless of the presence of symptoms and despite clinical success of therapy, WB-MRI demonstrates typical CRMO bone lesions at longterm followup. Similar to the discordance at initial encounters, even in patients with symptomatic sites, additional bone lesions are present at sites that are asymptomatic. Although lesions are no longer symptomatic under or after therapy, the inflammation may persist, rendering them clinically undetectable.

The authors are to be congratulated on their efforts and successful recruitment of patients to return for reevaluations after up to 26 years. The authors propose that " .... all patients with CRMO, including patients in clinical remission, require longterm clinical followup and should undergo evaluation with WB-MRI on a regular basis until radiological remission or a steady state of disease is achieved." While the definition and significance of "steady state of disease" in CRMO patients is unclear, it appears that the recommendation for WB-MRI surveillance in CRMO patients demands an answer to the following question raised by the results of their study: "What is the clinical and prognostic relevance of clinically undetectable CRMO lesions?"

\footnotetext{
JAN FRITZ, MD, PD, DARB

Johns Hopkins University School of Medicine, Russell H. Morgan Department of Radiology and Radiological Science,

601 N. Caroline St., JHOC 5168,

Baltimore, Maryland 21287, USA.
}

Address correspondence to Dr. Fritz. E-mail: jfritz9@jhmi.edu

\section{REFERENCES}

1. Giedion A, Holthusen W, Masel LF, Vischer D. [Subacute and chronic "symmetrical" osteomyelitis]. [Article in multiple languages] Ann Radiol (Paris) 1972;15:329-42.

2. Probst FP, Bjorksten B, Gustavson KH. Radiological aspect of chronic recurrent multifocal osteomyelitis. Ann Radiol (Paris) 1978;21:115-25.

3. Fritz J, Tzaribatchev N, Claussen CD, Carrino JA, Horger MS Chronic recurrent multifocal osteomyelitis: comparison of whole-body MR imaging with radiography and correlation with clinical and laboratory data. Radiology 2009;252:842-51.

4. von Kalle T, Heim N, Hospach T, Langendörfer M, Winkler P, Stuber T. Typical patterns of bone involvement in whole-body MRI of patients with chronic recurrent multifocal osteomyelitis (CRMO). Rofo 2013;185:655-61.

5. Roderick M, Shah R, Finn A, Ramanan AV. Efficacy of pamidronate therapy in children with chronic non-bacterial osteitis: disease activity assessment by whole body magnetic resonance imaging. Rheumatology 2014;53:1973-6.

6. Beck C, Morbach H, Beer M, Stenzel M, Tappe D, Gattenlöhner S, et al. Chronic nonbacterial osteomyelitis in childhood: prospective follow-up during the first year of anti-inflammatory treatment. Arthritis Res Ther 2010;12:R74.

7. Guerin-Pfyffer S, Guillaume-Czitrom S, Tammam S, Kone-Paut I. Evaluation of chronic recurrent multifocal osteitis in children by whole-body magnetic resonance imaging. Joint Bone Spine 2012;79:616-20

8. Fritz J, Tzaribachev N, Thomas C, Wehrmann M, Horger MS, Carrino JA, et al. Magnetic resonance imaging-guided osseous biopsy in children with chronic recurrent multifocal osteomyelitis. Cardiovasc Intervent Radiol 2012;35:146-53.

9. Voit AM, Arnoldi AP, Douis H, Bleisteiner F, Jansson MK, Reiser MF, et al. Whole-body magnetic resonance imaging in chronic recurrent multifocal osteomyelitis: clinical longterm assessment may underestimate activity. J Rheumatol 2015;42:1455-62.

J Rheumatol 2015;42:1359-60; doi:10.3899/jrheum.150676 